\title{
Osmotic and cryoprotective effects of glycerol-sucrose solutions on Day-3 mouse embryos
}

\author{
András Széll* and J. N. Shelton \\ Department of Immunology, The John Curtin School of Medical Research, Australian National \\ University, P.Q. Box 334, Canberra 2601, A.C.T., Australia
}

\begin{abstract}
Summary. The relative volume of Day-3 mouse embryos changed as a linear function of the reciprocal of osmolarity of non-permeating solutes after $10 \mathrm{~min}$ exposure to sucrose and glycerol-sucrose solutions at $20^{\circ} \mathrm{C}$. The slope of the linear regression line was less in glycerol-sucrose than in sucrose solutions because glycerol permeation caused re-expansion.

Before freezing by direct transfer to $-180^{\circ} \mathrm{C}$ the embryos were placed into glycerolsucrose in 1-step (1-step equilibration) or first into glycerol and then into glycerolsucrose (2-step equilibration). Using 2-step equilibration the post-thaw survival rate was substantially higher at 3.0 and $4.0 \mathrm{M}$-glycerol levels and less dependent on changes in the sucrose concentration within the range of 0.125 to $1.0 \mathrm{M}$ than with 1 -step equilibration. Under optimal conditions $90-95 \%$ of rapidly frozen embryos developed to blastocysts in vitro and $30 \%$ into live young in vivo.

It is suggested that the cryoprotective role of glycerol is due to its ability to reduce osmotic pressure differences between the extra and intracellular spaces during rapid freezing of embryos.
\end{abstract}

\section{Introduction}

Since the first methods for frozen storage of mouse embryos were developed by Whittingham et al. (1972) and by Wilmut (1972), further research has concentrated on the simplification of procedures, application to other species and improvement of viability. Amongst the most important advances in achieving these goals are the recognition that embryos survive rapid thawing if the slow rate freezing is terminated at -30 to $-45^{\circ} \mathrm{C}$ (Willadsen, 1977; Willadsen et al., 1978) and the development of an in-straw cryoprotectant dilution technique (Leibo et al., 1982; Renard et al., 1982).

Miyamoto \& Ishibashi (1983) reported that mouse embryos survive freezing by direct transfer to subzero temperatures without seeding. The viability of directly frozen embryos depends on the degree of dehydration which can be controlled by the inclusion of sucrose in the freezing medium (Nguyen et al., 1983; Renard et al., 1984).

The effects of cryoprotectant and sucrose concentrations on the survival of rapidly frozen embryos have been studied by several authors including Bui-Xuan-Nguyen et al. (1984), Vincent et al. (1985) and Biery et al. (1986). The present work examines the interrelationships between glycerol and sucrose concentrations in the freezing medium and the method of equilibration on the osmotic behaviour and post-thaw survival of rapidly frozen Day-3 mouse embryos.

*Permanent address: Mezögazdasági Föiskola, H-740I Kaposvár, Pf. 16, Hungary. 


\section{Materials and Methods}

\section{Source of embryos}

WEHI mice aged 6-12 weeks were induced to superovulate by intraperitoneal injections of 5 i.u. PMSG and 2 i.u. hCG $46 \mathrm{~h}$ apart. The females were paired with males at the time of hCG injection and the embryos were recovered 66-70 $\mathrm{h}$ later by flushing the reproductive tracts with Dulbecco's phosphate-buffered saline (PBS) supplemented with $5 \%$ fetal calf serum (FCS). PBS was prepared as described by Dulbecco \& Vogt (1954) except that $\mathrm{MgSO}_{4} \cdot 7 \mathrm{H}_{2} \mathrm{O}$ was used instead of $\mathrm{MgCl}_{2} \cdot 6 \mathrm{H}_{2} \mathrm{O}$ in equimolar concentration. Morphologically normal 8- to 16-cell embryos were washed in several changes of medium and randomly allocated to treatments.

\section{Studies on the osmotic effects of sucrose and glycerol-sucrose}

Experiments 1 and 2. The effect of sucrose concentration on the volume of embryos was studied in sucrose and in glycerol-sucrose solutions. The volume of embryos was measured after $10 \mathrm{~min}$ exposure at $20^{\circ} \mathrm{C}$ to sucrose $(0 \cdot 125$, $0.25,0.5$ or $1.0 \mathrm{M}$; Exp. 1) and glycerol-sucrose ( $3.0 \mathrm{M}$-glycerol $+0,0.125,0.25,0.5$ or $1.0 \mathrm{M}$-sucrose; Exp. 2) solutions. In both experiments the experimental unit was 1 embryo and there were 6 replications.

Experiment 3. The effects of permeation by glycerol and dehydration by sucrose on the osmotic behaviour of embryos in glycerol-sucrose were studied. The embryos were dehydrated by $0.25 \mathrm{M}$-sucrose $(10 \mathrm{~min})$, permeated by $4.0 \mathrm{M}$-glycerol $(10 \mathrm{~min})$ or left in PBS $+5 \%$ FCS before equilibration in $4.0 \mathrm{M}$-glycerol $+0.25 \mathrm{M}$-sucrose at $20^{\circ} \mathrm{C}$. The volume of the same embryos was measured after $1 \cdot 25,2 \cdot 5,5,10$ and 20 min exposure to glycerol-sucrose. In Exp. 3 the experimental unit was 1 embryo and the experiment had 5 replications.

Measurement of changes in the volume of embryos. The volume of embryos was measured as described in detail by Jackowski et al. (1980) and Széll \& Shelton (1986b). The embryos were pipetted into large pools of experimental solutions, prepared in PBS $+5 \%$ FCS, and then into a flat drop of identical medium which was covered by oil to stop evaporation. Photographs were taken at predetermined times through an inverted microscope and the volume of embryos was estimated from their cross-sectional area assuming a spherical shape. Eight- to 16-cell mouse embryos well approximated the spherical shape because of the regular arrangement of blastomeres and the compaction of the embryo itself. The volume of embryos in experimental solutions was expressed as a percentage of their isosmotic volume.

\section{Studies on the viability of frozen-thawed embryos}

Experiment 4. The effect of the method of equilibration on the post-thaw survival of embryos was studied. The embryos were exposed to $0.5 \mathrm{M}$-sucrose or to $4.0 \mathrm{M}$-glycerol for $5 \mathrm{~min}$ before being pipetted into $4.0 \mathrm{M}$-glycerol +0.5 $\mathrm{M}$-sucrose for another $5 \mathrm{~min}$ or were placed into $4.0 \mathrm{M}$-glycerol $+0.5 \mathrm{M}$-sucrose in 1 -step for $10 \mathrm{~min}$ and then frozen. The experimental unit was a group of 20 embryos and the experiment had 3 replications. The number of frozen-thawed embryos developing to blastocysts was expressed as a proportion of those frozen.

Experiments 5 and 6 . The effects of glycerol $(2.0,3.0,4.0$ or $5.0 \mathrm{M})$ and sucrose $(0,0.125,0.25,0.5$ or $1.0 \mathrm{M})$ concentrations of the freezing medium on the post-thaw survival were studied. In Exp. 5 the embryos were placed into glycerol-sucrose solutions for 10 min directly from PBS $+5 \%$ FCS (1-step addition). In Exp. 6, before placing into glycerol-sucrose for $5 \mathrm{~min}$, the embryos were exposed for $5 \mathrm{~min}$ to a solution containing glycerol only in identical concentration (2-step addition). After equilibration the embryos were frozen. The experimental unit was a group of 20 embryos and Exp. 5 had 5 and Exp. 6 had 3 replications. The number of frozen-thawed embryos developing to blastocysts was expressed as a proportion of those frozen.

Experiment 7 . The effects of freezing and in-vitro culture on the subsequent development of embryos in foster mothers were studied. According to random allocation to treatment, groups of 10 fresh or frozen-thawed embryos were transferred into recipients after $24 \mathrm{~h}$ culture in Whitten's medium or without culture. Before freezing the embryos were equilibrated in $4.0 \mathrm{M}$-glycerol $+0.5 \mathrm{M}$-sucrose in 2-step as described at Exp. 6. Five embryos were transferred into each uterine horn of recipients on the afternoon of the third day of pseudopregnancy. The recipients were allowed to go to term. The number of live-born young was expressed as a proportion of embryos allocated to treatment (overall survival rate). The experimental unit was a group of 10 embryos and the experiment had 6 replications.

Freezing and thawing procedures. The embryos were exposed to freezing media at $20^{\circ} \mathrm{C}$ and then frozen in $0.5 \mathrm{ml}$ plastic straws as described in detail by Széll \& Shelton (1986a). The straws contained $440 \mu \mathrm{l} 1 \cdot 0 \mathrm{M}$-sucrose solution and $40 \mu \mathrm{l}$ freezing medium separated by an air bubble. All experimental solutions were prepared in PBS $+5 \%$ FCS. Groups of 20 (Exps 4, 5 and 6) or 10 (Exp. 7) embryos were pipetted into the freezing medium fraction of the straws. The straws were frozen by direct transfer into liquid nitrogen vapour (about $-180^{\circ} \mathrm{C}$ ) and then stored in liquid nitrogen for 5 days or longer. After thawing in a water bath at $35^{\circ} \mathrm{C}$ the glycerol was removed by in-straw sucrose dilution (Leibo et al., 1982; Renard et al., 1982). After mixing the two fractions of the straw, 10 min at $20^{\circ} \mathrm{C}$ was allowed for glycerol removal (Széll \& Shelton, 1986a). The embryos were washed in PBS $+5 \%$ FCS and cultured for 2 days in Whitten's medium. 


\section{Statistical analysis}

In Exps 1 and 2 the regression of the relative volume of embryos on the reciprocal of osmolarity of non-permeating solutes was calculated by the method of least squares. The significance of unusual deviations from the regression lines were tested by Student's $t$ test. The difference between the slopes of the regression lines and the results of Exps 3, 4, 5 and 6 were evaluated by analysis of variance. Experiment 3 was of split-plot design in which measurements on the same embryo at different times constituted the subplots. The number of embryos developing to blastocysts in culture (Exps 4, 5 and 6) was transformed into angles for analysis. In Exps 4, 5 and 6 the treatment effects were tested against the theoretical error variance unless the experimental error significantly exceeded the theoretical estimate. The significance of differences amongst treatment means was tested by orthogonal comparisons.

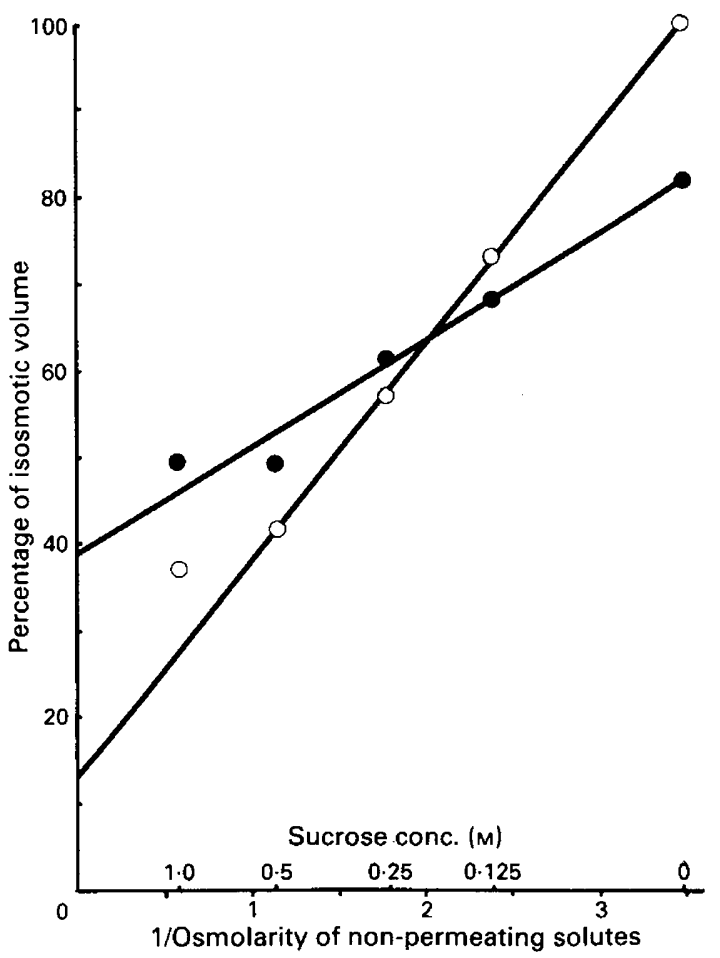

Fig. 1. Percentage of isosmotic volume of Day-3 mouse embryos as a function of the reciprocal of calculated osmolarity created by non-permeating solutes. The embryos were exposed to sucrose $(\mathrm{O}-\mathrm{O})$ or to $3.0 \mathrm{M}$-glycerol + sucrose $\left(-\right.$ ) solutions at $20^{\circ} \mathrm{C}$ for $10 \mathrm{~min}$. Linear regression lines were fitted by the method of least squares. Each point is the mean value for 6 embryos (Exps 1 and 2).

\section{Results}

\section{Osmotic response of embryos to glycerol-sucrose}

Effect of sucrose concentration on the volume of embryos. In accord with the modified Boylevan't Hoff equation there was a linear relation between the relative volume of embryos and the reciprocal of the calculated osmolarity of non-permeating solutes in sucrose and glycerol-sucrose solutions (Fig. 1). The linear regression lines were fitted by the method of least squares (Exp. 1, $y=13.09+24.98 x$; Exp. $2, y=39.45+12.02 x$; where $y$ is the relative volume of the embryos and $x$ is the reciprocal of the osmolarity of non-permeating solutes). The deviation from the shrinkage 
predicted by the regression line was unusually high in $1.0 \mathrm{M}$-sucrose $(P=0.038)$ therefore this point was omitted from the calculation. The slope of the regression line for embryos exposed to sucrose was significantly steeper than for those exposed to glycerol-sucrose $(P<0.001)$. The correlation between the relative volume of embryos and the reciprocal of the osmolarity of non-permeating solutes was close in both experiments (Exp. 1, $r=0.999$; Exp. 2, $r=0.985$ ).

Effect of previous exposure to sucrose or glycerol on the osmotic behaviour of embryos in glycerolsucrose. The results are shown in Fig. 2. The pattern of changes in the volume of embryos was dependent on the pretreatment $(P<0.001)$. The volume of embryos previously dehydrated was smaller than those without pretreatment and both increased with the time of equilibration. The volume of embryos previously permeated by glycerol was initially the highest and showed a decrease with the time of equilibration.

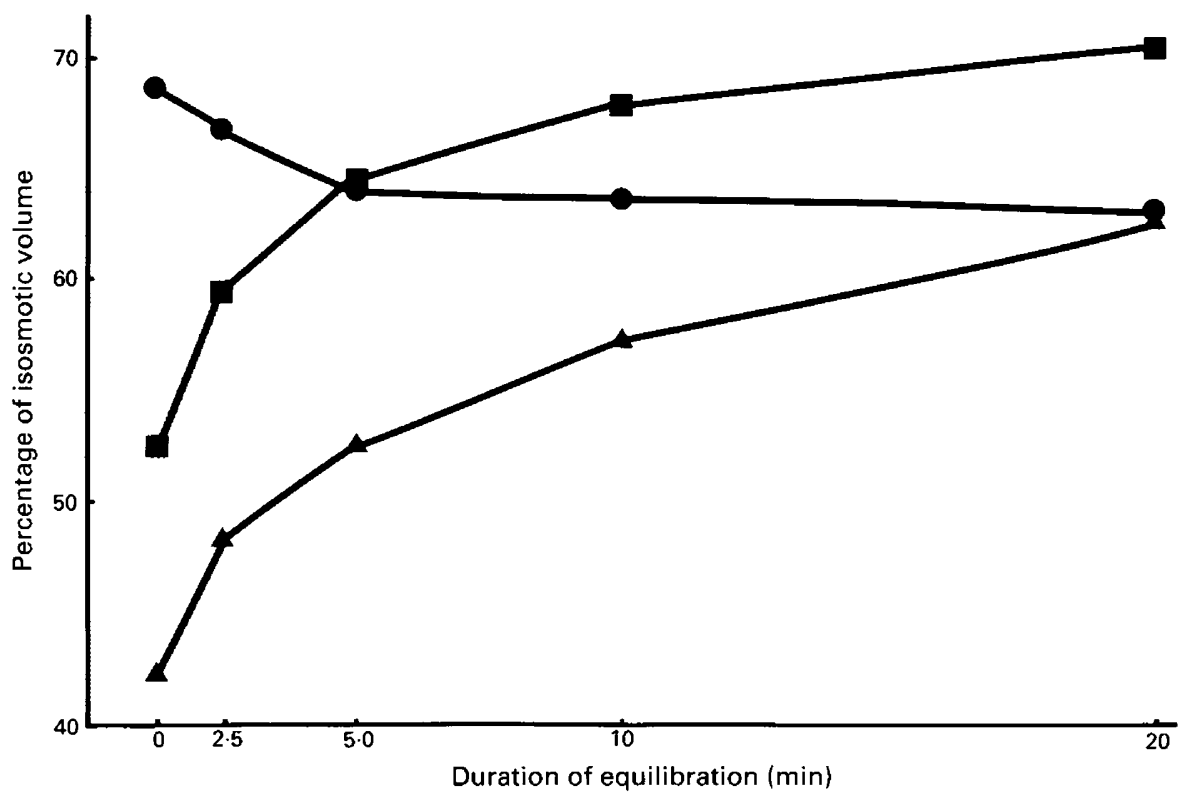

Fig. 2. Effects of previous exposure to $0.25 \mathrm{M}$-sucrose $(\Delta)$ or $4 \cdot 0 \mathrm{M}$-glycerol $(\bullet)$ on the osmotic behaviour of Day-3 mouse embryos in $4.0 \mathrm{M}$-glycerol $+0.25 \mathrm{M}$-sucrose at $20^{\circ} \mathrm{C}$. Five embryos were exposed to pretreatments or left without pretreatment $(\boldsymbol{\square})$ and the volume of the same embryos was measured during equilibration in glycerol-sucrose (Exp. 3).

Viability of embryos in vitro after rapid freezing in glycerol-sucrose

Effect of method of equilibration on the survival of frozen-thawed embryos in vitro. The survival rate of embryos which were previously dehydrated by sucrose, permeated by glycerol or placed into glycerol-sucrose in 1 -step was 53,74 and $58 \%$ respectively. The viability of previously permeated embryos was significantly $(P>0.001)$ higher than that of the other two groups.

Effect of glycerol and sucrose concentrations on the viability of frozen-thawed embryos in vitro. The survival of rapidly frozen embryos as a function of glycerol and sucrose concentrations of the freezing medium was studied after 1-step and 2-step addition (Table 1). Visual observation indicated that freezing media containing $4.0 \mathrm{M}$-glycerol $+1.0 \mathrm{M}$-sucrose, $5.0 \mathrm{M}$-glycerol +0.5 or $1.0 \mathrm{M}$-sucrose vitrified upon transfer into liquid nitrogen vapour. At 3.0 and $4.0 \mathrm{M}$-glycerol concentration the survival rate for most treatment combinations was higher using 2-step addition 
Table 1. Effects of glycerol and sucrose concentration in the freezing medium on the percentage of embryos surviving rapid freezing after equilibration in 1-step (Exp. 5) or in 2-steps (Exp. 6)

\begin{tabular}{|c|c|c|c|c|c|c|c|c|}
\hline \multirow[b]{2}{*}{ Exp. } & \multirow{2}{*}{$\begin{array}{l}\text { Method of } \\
\text { equilibration }\end{array}$} & \multirow{2}{*}{$\begin{array}{l}\text { Glycerol } \\
\text { conc. (M) }\end{array}$} & \multicolumn{5}{|c|}{ Sucrose conc. (M) } & \multirow[b]{2}{*}{ Mean } \\
\hline & & & 0 & $0 \cdot 125$ & $0 \cdot 25$ & 0.5 & 1.0 & \\
\hline 5 & I-step & $\begin{array}{l}2 \cdot 0 \\
3 \cdot 0 \\
4.0 \\
5 \cdot 0\end{array}$ & $\begin{array}{c}7^{\mathrm{ab}} \\
47^{\mathrm{bc}} \\
56^{\mathrm{b}} \\
50^{\mathrm{a}}\end{array}$ & $\begin{array}{l}14^{\mathrm{b}} \\
57^{\mathrm{c}} \\
62^{\mathrm{b}} \\
67^{\mathrm{b}}\end{array}$ & $\begin{array}{l}10^{\mathrm{b}} \\
60^{\mathrm{c}} \\
68^{\mathrm{bc}} \\
82^{\mathrm{c}}\end{array}$ & $\begin{array}{c}1^{\mathrm{a}} \\
30^{\mathrm{b}} \\
76^{\mathrm{c}} \\
95^{\mathrm{d}}\end{array}$ & $\begin{array}{r}0^{\mathrm{a}} \\
3^{\mathrm{a}} \\
28^{\mathrm{a}} \\
78^{\mathrm{c}}\end{array}$ & $\begin{array}{r}6 \\
39 \\
58 \\
74\end{array}$ \\
\hline 6 & 2-step & $\begin{array}{l}2 \cdot 0 \\
3 \cdot 0 \\
4 \cdot 0 \\
5 \cdot 0\end{array}$ & $\begin{array}{r}0^{\mathrm{a}} \\
23^{\mathrm{a}} \\
45^{\mathrm{a}} \\
63^{\mathrm{a}}\end{array}$ & $\begin{array}{c}5^{\mathrm{a}} \\
78^{\mathrm{c}} \\
62^{\mathrm{b}} \\
78^{\mathrm{ab}}\end{array}$ & $\begin{array}{l}40^{b} \\
85^{c} \\
93^{c} \\
82^{b}\end{array}$ & $\begin{array}{r}3^{\mathrm{a}} \\
68^{\mathrm{b}} \\
83^{\mathrm{c}} \\
92^{\mathrm{b}}\end{array}$ & $\begin{array}{r}0^{\mathrm{a}} \\
48^{\mathrm{b}} \\
93^{\mathrm{c}} \\
92^{\mathrm{b}}\end{array}$ & $\begin{array}{l}10 \\
61 \\
75 \\
81\end{array}$ \\
\hline
\end{tabular}

The number of embryos per treatment combination was 100 in Exp. 5 and 60 in Exp. 6. Means within the same row with different superscripts are significantly different $(P<0.01)$.

compared to 1-step addition. In both experiments at all levels of sucrose the viability of embryos increased with higher glycerol concentrations. The maximum rate of survival was not different in the two experiments (about 90-95\%), but was achieved at lower glycerol concentration when 2-step equilibration was used $(4.0 \mathrm{M})$ compared to 1 -step equilibration $(5 \cdot 0 \mathrm{M})$.

In Exp. 5 the optimum concentration of sucrose depended on the level of glycerol; at $2 \cdot 0,3 \cdot 0$, 4.0 and $5.0 \mathrm{M}$-glycerol concentration the highest survival was obtained when the freezing medium contained $0 \cdot 125,0 \cdot 25,0.5$ and $0.5 \mathrm{M}$-sucrose respectively. At all glycerol concentrations the viability decreased at sucrose levels higher than optimal.

In Exp. 6 the viability was less dependent on the sucrose concentration; at all glycerol levels the optimal survival rate was attained at $0.25 \mathrm{M}$-sucrose concentration and in 4.0 and $5.0 \mathrm{M}$-glycerol solutions there was no decline with higher sucrose concentrations.

Table 2. Effects of rapid freezing and in-vitro culture on the development of Day-3 embryos to live young in recipients (Exp. 7)

\begin{tabular}{|c|c|c|c|c|}
\hline \multicolumn{2}{|c|}{ Treatment of embryos } & \multirow{2}{*}{$\begin{array}{l}\text { Recipients } \\
\text { giving birth }\end{array}$} & \multirow{2}{*}{$\begin{array}{c}\text { Mean } \\
\text { litter size }\end{array}$} & \multirow{2}{*}{$\begin{array}{l}\text { Overall survival } \\
\text { rate of embryos* }\end{array}$} \\
\hline Freezing & Culture & & & \\
\hline Frozen & $\begin{array}{l}\text { Not cultured } \\
\text { Cultured }\end{array}$ & $\begin{array}{l}3 / 6 \\
5 / 6\end{array}$ & $\begin{array}{l}3 \cdot 3 \\
3 \cdot 6\end{array}$ & $\begin{array}{l}17 \% \\
30 \%\end{array}$ \\
\hline Fresh & $\begin{array}{l}\text { Not cultured } \\
\text { Cultured }\end{array}$ & $\begin{array}{l}4 / 6 \\
5 / 6\end{array}$ & $\begin{array}{l}4 \cdot 0 \\
4 \cdot 6\end{array}$ & $\begin{array}{l}27 \% \\
38 \%\end{array}$ \\
\hline
\end{tabular}

*The number of embryos per treatment combination was 60 .

Viability of embryos in vivo after rapid freezing in glycerol-sucrose

The effects of rapid freezing and $24 \mathrm{~h}$ in-vitro culture on the proportion of recipients giving birth, their mean litter size and the overall survival rate of embryos are summarized in Table 2 . The proportion of frozen-thawed embryos developing to term was less than that of fresh embryos. In-vitro culture improved the overall survival rate of frozen-thawed embryos from 17 to $30 \%$; there was much less difference betwen the overall survival rates of fresh embryos transferred without or after culture $(27 \%$ vs $38 \%)$. 


\section{Discussion}

The osmotic response of mouse embryos to permeating solutes, non-permeating solutes and to the mixtures of permeating and non-permeating solutes have been described by Jackowski et al. (1980), Leibo (1980) and Széll \& Shelton (1986b). In the solutions of non-permeating solutes like sucrose the osmotic equilibrium is established by shrinkage and there is no further change afterwards. In glycerol and in glycerol-sucrose solutions, however, glycerol permeation continues after the establishment of osmotic equilibrium as demonstrated by the re-expansion of embryos (Jackowski et al., 1980; Széll \& Shelton, 1986b).

The measurements of the osmotic response were based on the assumption that the shape of Day-3 mouse embryos is spherical. The validity of this assumption is proved by the close correlation between the relative volume of embryos and the reciprocal of the osmotic pressure. Osmotic pressure created by glycerol was not considered in Fig. 1 because at $20^{\circ} \mathrm{C}$ after $10 \mathrm{~min}$ exposure to glycerol-sucrose the embryos become almost fully permeated by glycerol (Széll \& Shelton, 1986b). This presentation also made the osmotic response of the embryos to the same sucrose concentration in sucrose and in glycerol-sucrose solutions readily comparable. The embryos responded osmotically to increases in sucrose concentration in both solutions by reducing their volume. The deviation from the predicted shrinkage in $1.0 \mathrm{M}$-sucrose indicates that embryos do not respond to very high osmotic pressures like 'perfect osmometers'. This deviation means the development of an osmotic pressure gradient between the extra- and intracellular spaces. Exceeding a tolerable limit in this pressure difference will inevitably damage the cell membrane. Such osmotic damage may explain the toxic effects of highly concentrated sucrose solutions (Széll \& Shelton, 1986a). Similar deviations from the predicted volumetric response were found for erythrocytes when the osmolarity of non-permeating solutes exceeded 1.1 osmol (Meryman, 1968). Red cell ghosts which have no cellular contents, but whose membrane characteristics are still normal show no departure from the predicted osmometric response. This suggests that deviations in normal cells are caused by the cellular contents (Meryman, 1970). Extrapolation of the regression line of Exp. 1 to infinitely high osmolality indicates that about $13 \%$ of the isosmotic volume of Day-3 mouse embryos is nonsolvent volume. Slightly higher estimates have been made for fertilized mouse ova and for bovine embryos by Leibo $(1980,1986)$.

The smaller slope of the regression line for embryos exposed to glycerol-sucrose than for those exposed to sucrose can be explained by re-expansion in glycerol-sucrose. In glycerol-sucrose solution the extracellular osmotic pressure is counteracted not only by shrinkage but also by intracellular glycerol. Therefore, at sucrose concentrations of $0.25 \mathrm{M}$ or higher less shrinkage was required to maintain osmotic equilibrium in glycerol-sucrose than in sucrose solutions.

The requirement for higher glycerol concentrations during rapid freezing compared to controlled rate freezing cannot be explained by protection against solution effects since the exposure to such effects is much shorter (Széll \& Shelton, 1986b). Meryman (1970) suggested that the injury of dehydrated cells during freezing is not caused by specific effects of salts (solution effects), but by the increased osmolarity of non-permeating solutes in the extracellular space. The osmolarity of nonpermeating solutes increases to $5.0 \mathrm{osmol}$ (about 17 times the isosmotic value) at $-20^{\circ} \mathrm{C}$ (Rall et al., 1978; Mazur, 1980). The present experiments suggest that embryos, like erythrocytes, are unable to maintain osmotic equilibrium with very concentrated solutions and an osmotic pressure difference will damage the cell membrane. Glycerol decreases the extracellular osmolarity of non-permeating solutes due to its ability to reduce the frozen fraction of water (Rall et al., 1978) and increases the intracellular osmolarity through permeation. This suggests that glycerol protects embryos during rapid freezing through reducing the osmotic pressure difference between the extraand intracellular spaces. A corollary to this explanation is that glycerol must permeate the cells to exert its protective effect through increasing the intracellular osmolarity. The present results, which show that previous exposure to sucrose reduced the rate of glycerol permeation and resulted in lower post-thaw survival rate compared to no pretreatment, support this proposition. 
The differences between the post-thaw survival of embryos after 1-step and 2-step addition are probably also related to alterations in glycerol permeation. The survival rate of embryos frozen in media containing 3.0 or $4.0 \mathrm{M}$-glycerol was substantially higher and less dependent on sucrose concentration using 2-step rather than 1-step addition. The optimum survival rate was also attained at a lower glycerol level when the embryos were placed first into glycerol and then into glycerol-sucrose. The concentrations of sucrose and glycerol in the freezing medium were in interaction; the optimal results were obtained at higher sucrose concentrations with increasing glycerol level. Biery et al. (1986) found a similar relationship between sucrose and glycerol concentrations for rapidly frozen Day-4 mouse embryos.

Our previous work (Széll \& Shelton, 1986a) showed that $0.5 \mathrm{M}$-sucrose is adequate for the dilution of up to $4.0 \mathrm{M}$ concentrations of glycerol. It was therefore assumed that in the present experiments the dilution of 2.0 to $5.0 \mathrm{M}$-glycerol with $1.0 \mathrm{M}$-sucrose did not cause any osmotic damage.

The occurrence of vitrification in contentrated glycerol-sucrose solutions indicates that there is no need to use more complex media which require step-wise addition and removal at $4^{\circ} \mathrm{C}$ ( $\mathrm{Rall} \&$ Fahy, 1985) for the ice-free cryopreservation of mouse embryos. From the present experiments, however, it appears that there is no benefit offered by vitrification in addition to that provided by dehydration and the presence of a permeating cryoprotectant.

Under optimal conditions the in-vitro survival rate of frozen-thawed embryos (90-95\%) was almost identical with the viability of unfrozen embryos (90-99\%) (Széll \& Shelton, 1986a). The in-vivo viability of rapidly frozen and fresh embryos was different, indicating that rapid freezing causes substantial damage to embryos. This difference was less than that reported for embryos frozen at controlled rates (Whittingham et al., 1977; Rall et al., 1984). Whittingham et al. (1977) cultured frozen-thawed Day-3 mouse embryos before transferring them into recipients. The beneficial effect of short-term culture on subsequent in-vivo development of Day-4 embryos frozen at controlled rates was reported by Rall et al. (1984) and the present results confirm this for rapidly frozen Day-3 embryos.

We thank Ms Klára Széll for excellent technical assistance and Ms Rebecca Edmondson for the preparation of figures.

\section{References}

Biery, K.A., Seidel, G.E., Jr \& Elsden, R.P. (1986) Cryopreservation of mouse embryos by direct plunging into liquid nitrogen. Theriogenelogy 25, 140, Abstr.

Bui-Xuan-Nguyen, N., Heyman, Y. \& Renard, J.P. (1984) Direct freezing cattle embryos after partial dehydration at room temperature. Theriogenology 22, 389-399.

Dulbecco, R. \& Vogt, M. (1954) Plaque formation and isolation of pure lines with poliomyelitis viruses. $J$. exp. Med. 99, 167-182.

Jackowski, S., Leibo, S.P. \& Mazur, P. (1980) Glycerol permeabilities of fertilized and unfertilized mouse ova. J. exp. Zool. 212, 329-341.

Leibo, S.P. (1980) Water permeability and its activation energy of fertilized and unfertilized mouse ova. $J$. Membrane Biol. 53, 179-188.

Leibo, S.P. (1986) Cryobiology: preservation of mammalian embryos. In Genetic Engineering of Animals: an Agricultural Perspective, pp. 251-272. Eds J. W. Evans \& A. Hollaender. Plenum Press, New York.
Leibo, S.P., West, A.W., III \& Perry, B. (1982) A one-step method for direct nonsurgical transfer of frozen-thawed bovine embryos. II. Applied studies. Cryobiology 19, 674, Abstr.

Mazur, P. (1980) Fundamental aspects of the freezing of cells, with emphasis on mammalian ova and embryos. Proc. 9th Int. Congr. Anim. Reprod. \& A.I., Madrid I, 99-114.

Meryman, H.T. (1968) Modified model for the mechanism of freezing injury in erythrocytes. Nature, Lond. 218, 333-336.

Meryman, H.T. (1970) The exceeding of a minimum tolerable cell volume in hypertonic suspensions as a cause of freezing injury. In The Frozen Cell (Ciba Fdn Symp.), pp. 51-67. Eds G. E. W. Wolstenholme \& M. O’Connor. J. \& A. Churchill, London.

Miyamoto, H. \& Ishibashi, T. (1983) Solid $\mathrm{CO}_{2}$ freezing of mouse embryos. J. Reprod. Fert. 67, 107-111.

Nguyen, B.X., Renard, J.P. \& Garnier, V. (1983) Rapid freezing and thawing of 2-cell-stage rabbit embryos 
after partial dehydration at room temperature. Cryobiology 20, 742, Abstr.

Rall, W.F. \& Fahy, G.M. (1985) Ice-free cryopreservation of mouse embryos at $-196^{\circ} \mathrm{C}$ by vitrification. Nature, Lond. 313, 573-575.

Rall, W.F., Mazur, P. \& Souzu, H. (1978) Physicalchemical basis of the protection of slowly frozen human erythrocytes by glycerol. Biophys. J. 23, $101-120$.

Rall, W.F., Wood, M.J. \& Kirby, C. (1984) The transfer of frozen-thawed mouse embryos in dimethyl sulfoxide: effect of dilution and short-term culture. Cryobiology 21, 710-711, Abstr.

Renard, J.-P., Heyman, Y. \& Ozil, J.-P. (1982) Congélation de l'embryon bovin: une novelle méthode de décongélation pour le transfer cervical d'embryons conditionnés une seule fois en paillettes. Annls Méd. Vét. 126, 23-32.

Renard, J.-P., Bui-Xuan-Nguyen \& Garnier, V. (1984) Two-step freezing of two-cell rabbit embryos after partial dehydration at room temperature. J. Reprod. Fert. 71, 573-580.

Széll, A. \& Shelton, J.N. (1986a) Sucrose dilution of glycerol from mouse embryos frozen rapidly in liquid nitrogen vapour. J. Reprod. Fert. 76, 401-408.

Széll, A. \& Shelton, J.N. (1986b) Role of equilibration before rapid freezing of mouse embryos. $J$. Reprod. Fert. 78, 699-703.
Vincent, C., Heyman, Y., Garnier, V., Smorag, Z. \& Renard, J.P. (1985) In vitro survival of early-stage rabbit and cow embryos directly frozen to intermediate temperature $\left(-25\right.$ to $\left.-30^{\circ} \mathrm{C}\right)$ before plunging into liquid nitrogen. Theriogenology 23, 234, Abstr.

Whittingham, D.G., Leibo, S.P. \& Mazur, P. (1972) Survival of mouse embryos frozen to $-196^{\circ}$ and $-269^{\circ} \mathrm{C}$. Science, N.Y. 178, $411-414$.

Whittingham, D.G., Lyon, M.F. \& Glenister, P.H. (1977) Long-term storage of mouse embryos at $-196^{\circ} \mathrm{C}$ : the effect of background radiation. Genet. Res., Camb. 29, 171-181.

Willadsen, S.M. (1977) Factors affecting the survival of sheep embryos during deep-freezing and thawing. In The Freezing of Mammalian Embryos (Ciba Fdn Symp. No. 52), pp. 175-189. Eds K. Elliott \& J. Whelan. Elsevier/North Holland, Amsterdam.

Willadsen, S.M., Polge, C. \& Rowson, L.E.A. (1978) In vitro storage of cattle embryos. In Control of Reproduction in the Cow, pp. 427-436. Ed. J. R. Sreenan. Martinus Nijhoff, Amsterdam.

Wilmut, I. (1972) The effect of cooling rate, warming rate, cryoprotective agent and stage of development on survival of mouse embryos during freezing and thawing. Life Sci. 11, 1071-1079.

Received 16 October 1986 